\title{
Information Flows in Future Advanced Manufacturing Ecosystems
}

\author{
Minna Lanz, Matti Majuri, and Reijo Tuokko \\ Tampere University of Technology, \\ Korkeakoulunkatu 10, 33720 Tampere, Finland \\ \{minna.lanz,matti.majuri,reijo.tuokko\}@tut.fi \\ http://www.tut.fi/tte
}

\begin{abstract}
Manufacturing is the backbone of each and every society, and in order for society to sustain in long run the manufacturing has to be sustainable as well. The sustainability in the field of manufacturing has traditionally been discussed in a sense of operational efficiency and environmental metrics. Rarely the link between individuals working in the company and the efficiency of operations has been established and discussed deeply. This link is information flow that combines both tacit and formal information in a dynamically changing socio-technical environment. In this paper the information flow between individuals in different levels of company hierarchy is utilized as the observation baseline. This paper discusses the information flow within a company and outlines sociotechnical challenges needed to solve in order to realize future Advanced Manufacturing Ecosystems.
\end{abstract}

Keywords: knowledge management, semantic information, tacit knowledge, systems thinking, social capital, know-how.

\section{Introduction}

Regarding the information flow two major challenges are identified: content of information and dynamic environment where the information is temporally valid. Today's design and operation control systems produce a variety of data. However, the sheer amount of data that lacks the structure, connectivity and intent cannot be utilized efficiently later on. There is ever the greater need to transfer this unstructured mass of bits and bytes to meaningful information and to human understandable knowledge, as it is understood in the industry. However there are major challenges in understanding the actual content of information flow. Today the information flow should connect the different design departments and activities to be part of the life-time information of a product including its processes and services. For example modern computer-aided design (CAD) tools has made it easier and faster to design products made up of more and more complicated and intricate parts. Without sufficient experience or guidance, todays designer runs the risk of developing product designs that are unnecessarily difficult to produce [14, 12. Another example of disruptions in information flow 
and/or understanding its content is vividly explained by Redman [16. He stated that quality errors in manufacturing relating to low quality data may raise costs up to $30 \%$. Furthermore, Redmans study indicates that $8-12 \%$ revenue losses occur because of data quality issues. Informally, 40-60\% of the expenses in service sector may be consumed as a result of poor data.

It is recognized that environment evolves and changes over the time and the system itself evolves, sometimes unpredictably, during its lifecycle. In recent years changes in the environment have become faster, stronger and harder to predict than ever before. Same time the manufacturing is becoming more decentralized causing challenges in the network level operations management. In order to operate in such dynamic and complex environment best of the both worlds; human intelligence and computers computing power are needed to be combined more sufficient manner. As the unpredictable changes occur, detailed simulations of large systems will become unreliable. Algorithms cannot solve the problems that arise, thus applying human intelligence to solve complex problems or choosing optimal scenario becomes highly important. Paradoxically the current operation strategy has forced human to be the processing unit while computer attempts to make intelligent decision over relatively vague input information. This strategy may improve the effectiveness of manufacturing in limited range of situations but on the other hand it definitely reduces the flexibility and agility of the manufacturing system.

Current engineering and production management methodologies and tools have been created for the old markets where the mass-customization was a norm. The IT-tools for these solutions offer proprietary services and interfaces. This paralyses healthy ecosystem growth, emergence of new start-ups, development and neglects the needs of agile and innovative SMEs. Contemporary tools and services, such as cloud-computing, emphasize on technology in favor of actual industry specific business needs. As a consequence ICT-centric solutions tend to help only in communication, but transfer existing (tedious) work practices in the new media. The current solutions are largely developed from the one perspective respecting the constraints from legacy ICT, not from the modern engineering business processes.

The challenges mentioned above are no doubt look very technical issues. However, technology itself cannot solve the issues. It is very likely that the technologyoriented approach will not be sufficient for the future advanced manufacturing ecosystems. This paper discusses of technical information management, tacit knowledge and system interoperation and relations. By understanding the technical possibilities, social know-how and how human-machine systems interoperate the advanced manufacturing ecosystems can emerge.

\section{Considering the Surrounding Systems}

\subsection{Semantics}

The main problem is that the models and documents created with different design and operation management systems are that the documents are meaningless 
from the knowledge point of view for systems other than their authoring system. The second problem is that the Design Intent is a concept that is not captured by the modern design systems. This raises even bigger challenges for the future manufacturing systems that require this information to provide autonomous reasoning. A case study done in Finnish manufacturing sector highlighted the challenge in capturing the design intent and managing the information flow among various stakeholders 8 . The case study was conducted in a large and globally operating manufacturing company, which possessed large network of subcontractors. The study revealed, that workers personality, human communication, and bureaucracy have a significant effect on what actually gets saved into the information systems, and furthermore what can be retrieved from the system later on. Furthermore the study suggested that the design intent was lost very early from the information content and due to that several important issues were constantly ignored. Different people also have very different ways of doing and understanding things, which may lead to misunderstandings in cooperation.

Knowledge builds on data and/or information that is organized and processed to convey understanding, experience, accumulated learning and expertise. In order to enhance the effectiveness, agility and reactivity of future the manufacturing systems it is needed to look at the detail level; more precisely, where the numbers are coming and how valid those are in given context. On the product design perspective this means that information flow content actually carries the design intent and it can be understood in a context of use. In the manufacturing operations level the simulation results are tied to the given context and are valid in that context. In the recent years industrial standards are being defined in a more computer-readable form, most notably since the emergence of eXtensive Mark-Up Language (XML) -based formats and computing power. XML as a language has number of advantages for developers and implementers. Structural language allows the compilation of specifications, creation of datasets and certain kinds of testing can be performed more easily. However, along the way XML markups have been used as a substitute for modeling the information - a dangerous shortcut that only works in communities that already share a common understanding of the meaning and usage of terms [11, [15].

Knowledge modeling and representation always reflect the view of the modelers on the domain to be modeled, although the ideal should be to initially remain as independent as possible of any purpose or application [17. More and more information will be retrieved from diverse sources originating from different circumstances and application. Therefore the resultant data are inherently built on different languages and representations that will put high demand on semantic integration solutions. Also, information will be fetched from diverse locations (over the global networks), which will be grounds for extensive use of web technologies. These two basic requirements (semantic integration, access over web) speaks for the adoption of semantic web technologies to describe and integrate (or perhaps rather inter-link) digital content on built environment. Semantic information enables the creation of built-in intelligence into versatile products. Real intelligent functions will be possible to be created based on semantic 
reasoning algorithms. The difference to "glued" intelligence is significant, because the product and the digital services related to products can utilize more detailed and specific information than traditionally [11].

\subsection{Systems Thinking}

Centralized systems based on hierarchical data share are giving a way to globally distributed production networks. These networks need to adapt rapidly to the production changes. Due to the resulting larger complexity of heterogeneous interacting components the system can no longer be only guided and controlled based on a human operator. Predicting the future state of dynamic system based on the static and often inaccurate or obsolete models of manufacturing system's state is not reliable or sufficient [10].

According to International Council of Systems Engineering (INCOSE) there is a growing awareness that the revised systems engineering approach is becoming a key to successfully design, develop and sustain the highly complex systems. However the field suffers from the lack of unified principles and models that support a wide range of domains [7. A shift away from a generic definition and application of systems engineering to a more specifically defined and precise application of systems engineering in diverse domains can thus be expected in the future. The future systems engineering environment will also fully support life cycle perspectives. In many respects, the future of systems engineering can be said to be model-based. A key driver will be the continued evolution of complex, intelligent, global systems that exceed the ability of the humans who design them to comprehend and control all aspects of the systems they are creating. The role of modeling will mature to respond to this need.

\subsection{Social Capital}

The role of humans in the Factories of the Future will be defined through the stimulation of working environments and conditions that bring about new forms of collaboration and interaction processes among humans, machines and the manufacturing technologies involved. Sustainability of the human capital, in Europe, is subject to the global economic conditions. Age and employment systems need to be adapted, while the societal impact of manufacturing on local environments ought to be taken into account, in terms of energy demands, life quality, natural resources and safety.

Lately, following to the well-known trends of globalization, transformation from vertical value chains to open value networks, web 2.0 and further developments, crowd-sourcing, dematerialization, virtualization etc., management theory has developed new approaches more coherent with the emergent business and social landscape, leveraging the human factor along with technology enablers. However, as the development advances with steady leaps it also causes pressures towards workforce. This pressure comes from dynamic and fast changes in organizational level, new technical solutions requiring faster learning curves and changes in global economy. In the recent years, analysis on employees wellbeing 
highlights that, more now than in the past, individuals have to deal with different objectives, desires, expectations and responsibilities, which can be clustered in two main categories of work and life. Bourne et al 3] stated that "dualcentric experience more overall satisfaction, greater work-life balance, and less emotional exhaustion", and that organizations should take care of employees as whole individuals in order to enhance their wellbeing.

According to EFFRA [4 manufacturing is evolving from being perceived as a production-centric operation to a human-centric business with greater emphasis on workers, suppliers and customers in-the-loop. ActionPlanT [1] envisions workers and managers alike given more opportunity for continuous development of skills and competences through novel knowledge delivery mechanisms. The human-centricity paradigms involve inclusion for young and elderly people Future enterprises will not only be better equipped for transferring skills to a new generation of workers but also proficient in assisting older workers with better user interfaces, intuitive user-experience-driven workflows and other aids, such as mobile and service robots.

\subsection{Technological Advances}

According to European Commission communication [5] a digital ecosystem is a self-organizing digital infrastructure aimed at creating a digital environment for networked organizations that supports the cooperation, the knowledge sharing, the development of open and adaptive technologies and evolutionary business models. Boley and Chang [2] proposed definition for a digital ecosystem by terms "open, loosely coupled, domain clustered, demand-driven, self-organizing agent environment", where each agent of each species is proactive and responsive regarding its own benefit/profit but is also responsible to its system. The digital ecosystem approach transposes the concepts to the digital world, reproducing the desirable mechanisms of natural ecosystems.

As several interacting natural ecosystems exist, several digital ecosystems exists due to differentiation and the development of endemic product and services tailored to specific local needs. The key enabling technologies developed within the digital ecosystem research aim at providing a knowledge- and service-oriented infrastructure that supports the spontaneous composition, distribution, evolution and adaptation of ICT-based services. This platform should allow the SME software industry to independently develop (and disseminate on the network) services and software components which will be composed forming complex, evolutive and adopted solutions. These technologies allow the spontaneous development and the cooperative provision of services and solutions, without the need for any keystone player, central coordination or central point of control/failure.

\section{Requirements for the Future Advanced Manufacturing Ecosystems}

The future advanced manufacturing ecosystems require new and more collaborative approaches, where the technical advantages are understood and accepted, 
where the social capital is appreciated and utilized and where the interactions of a whole system can be visualized and understood.

As the construction and development of an increasingly artifactual environment continue, little thought has been given to the coherence or compatibility of knowledge between different scientific disciplines. As a result, incompatibilities and contradictions have arisen within that environment, and, most ominously, between it and the surrounding natural environment 18. Yoshikawa (2008) introduced a term synthesiology. Synthesiology is a term for the theory of integration of scientific and technological knowledge from different disciplines with the needs of society.

McDonald (2011) has recommended that management should evolve to foster trust and teamwork; to create a fluid, flexible, customized work community environment; to decompose vertical organizational structures towards inter-intra organizational networks with emergent new roles for facilitators and brokers; to create new exciting, entertaining and challenging workplaces for young people 13. Kira et al (2008) emphasize that socially sustainable work organizations are able to both repeat accustomed and devise innovative solutions, and for this reason it is crucial for them to embrace individual stakeholders complexities and encourage them to cultivate it, while at the same time maintaining a holistic view of the organization [9]. Furthermore, Kira et al. [9] suggest that a higher level of collaborative shaping of work (with employee involvement) is highly conducive to sustainable work abilities and development of human resources.

As this paper focuses more on the information flow management in future Manufacturing Ecosystems, the collaboration, motivation and self-organizing interaction between individuals becomes highly important. Fischer [6] summarized well that in interpersonal communication the design intent is mostly casually conveyed and understood based on history, shared experience and circumstances. However, in this modern operation environment each stakeholder has a different context and different understanding of the problem. Communication breakdown occurs when the stakeholders have only little shared context. The message, design intent, gets lost in translation.

\section{Summary}

All in all the future interoperability among different stakeholders requires more multi-sectoral approach in order to tackle the arisen challenges. The efficient knowledge capture, processing, utilization and reuse as integral part of future product-services requires holistic approach, that includes understanding from following sector:

1. Social and economic sciences (network economics, community buildings, diffusion of knowledge and practices, legal aspects, business and organizational models),

2. System theory, self-organization of complex systems and epistemology

3. Computer science (mainly network architectures, semantic interoperability and formal languages) [5]. 
Only by adopting the holistic approach in design, operation management and manufacturing the new Advanced Manufacturing Ecosystems can emerge.

\section{References}

1. Majumdar, A., Szigeti, H.: ICT FOR MANUFACTURING - The ActionPlanT Vision for Manufacturing 2.0, Factories of the Future 2020 Roadmap. Consultation document. EFFRA - European Factories of the Future Research Association (2012)

2. Boley, H., Chang, E.: Digital Ecosystems: Principles and Semantics. In: IEEE International Conference on Digital Ecosystems and Technologies, Cairns, Australia (2007)

3. Bourne, K., Wilson, F., Lester, S.W., Kickul, J.: Embracing the whole individual: Advantages of a dual-centric perspective of work and life. Business Horizons 52(4), 387-398 (2009)

4. Industrial Advisory Group: Factories of the Future 2020 Roadmap, Consultation document. EFFRA European Factories of the Future Research Association (2012)

5. European Commission: Digital Ecosystems - the enabling technologies and paradigms for fostering endogenous local development, local capacity building and knowledge sharing processes providing tailored and personalized ICT services to citizens and business networks (2013), http://www.digital-ecosystems.org/

6. Fischer, G., Nakakojia, K., Ostwald, J.: Supporting the evolution of design artifacts with representations of context and intent. In: Proceedings of the 1st Conference on Designing Interactive Systems: Processes, Practices, Methods, and Techniques (1995)

7. Anon: Systems Engineering Vision 2020. International Council on Systems Engineering report, INCOSE-TP-2004-004-02 (September 2008)

8. Jarvenpaa, E., Lanz, M., Mela, J., Tuokko, R.: Studying the Information Sources and Flows in a Company - Support for the Development of New Intelligent Systems. In: Proceedings of Flexible Automation and Intelligent Manufacturing (2010)

9. Kira, M., Van Eijnatten, F.M.: Socially Sustainable Work Organizations: A Chaordic Systems Approach. Systems Research and Behavioral Science 25(6), 743$756(2008)$

10. Lanz, M., Nylund, H., Ranta, A., Luostarinen, P., Tuokko, R.: Set-up and first steps on capturing of realistic resource characteristics of an intelligent manufacturing environment. In: Proceedings of Flexible Automation and Intelligent Manufacturing (2010)

11. Lanz, M., Nykanen, O., Aaltonen, J., Ranta, P.A., Koskinen, K.T., Andersson, P.H.: Engineering Intelligence - Product-Service Concepts and Requirements in Industry. In: Proceedings of IEEE International Symposium of Assembly and Manufacturing, ISAM (2013)

12. Lohtander, M., Varis, J.: Collecting manufacturing information in a global distributed manufacturing environment. Mechanika 18(1), 84-88 (2012)

13. McDonald, P.: It's time for management version 2.0: Six forces redefining the future of modern management. Futures of Evolutionary Psychology 43(8), 797-808 (2011)

14. Pulkkinen, A., Rissanen, N., Vainio, V.: PLM state of the practice and future challenges in globally networked manufacturing companies. In: Proceedings of the 1st PDM Forum for Finland-Russia Collaboration, Lappeenranta, April 25-26 (2013)

15. Ray, S.R., Jones, A.: Manufacturing Interoperability. Journal of Intelligent Manufacturing 17, 681-688 (2006) 
16. Redman, T.C.: The impact of poor data quality on the typical enterprise. Communications of the ACM 41(2), 79-82 (1998), doi:10.1145/269012.269025

17. Spyns, P., Meersman, R.: Ontology Engineering and (Digital) Business Ecosystems: a case for a Pragmatic Web. IEEE (2007) ISBN 1-4244-0826 831-838

18. Yoshikawa, H.: Synthesiology as sustainability science. Integrated Research System for Sustainability Science 3, 169-170 (2008) 